\title{
Magnetically ordered and disordered sublattices in geometrically frustrated Ni chromite
}

\author{
N. Stüsser, M. Reehuis, M. Tovar, B. Klemke, A. Hoser, and J.-U- Hoffmann \\ Helmholtz-Zentrum Berlin für Materialien und Energie, D-14109 Berlin, Germany
}

The magnetic order/disorder of the Cr-moments in the antiferromagnetic (AF) state of the ferrimagnet $\mathrm{NiCr}_{2} \mathrm{O}_{4}$ is reinvestigated by neutron powder diffraction to clarify the observation of reduced $\mathrm{Cr}$ moments located on a pyrochlore like lattice [M. Reehuis et al., Phys. Rev. B 91, 024407 (2015)]. The change of the spin structure in the slightly $\mathrm{Cu}$-doped chromite $\mathrm{Ni}_{0.98} \mathrm{Cu}_{0.02} \mathrm{Cr}_{2} \mathrm{O}_{4}$ has been studied, where orthorhombic lattice distortions are stronger. For both chromites we observe at $2 \mathrm{~K}$ two magnetic phases: one with two propagation vectors $\boldsymbol{k}=$ $(0,0,1)$ and $\left(0,0, k_{z}\right)$, and a second one with $\boldsymbol{k}=(1 / 2,1 / 2,1 / 2)$. The latter phase disappears at $21 \mathrm{~K}$ for $\mathrm{NiCr}_{2} \mathrm{O}_{4}$ and at $24 \mathrm{~K}$ for $\mathrm{Ni}_{0.98} \mathrm{Cu}_{0.02} \mathrm{Cr}_{2} \mathrm{O}_{4}$. Data analysis shows that the two wave vectors can be assigned to two magnetic phases residing on two disparate $\mathrm{Cr}$ sublattices. These are identified by different chain directions of strongly bonded spin pairs along diagonals in the pseudo-tetragonal $a_{1} a_{2}$ plane. The less distorted $\mathrm{NiCr}_{2} \mathrm{O}_{4}$ shows the same subdivision of the lattice still with a saturated $\mathrm{Cr}$ moment for the $(0,0,1) /\left(0,0, k_{z}\right)$ phase, however, with a strongly reduced moment for the $(1 / 2,1 / 2,1 / 2)$ phase, whereas an almost saturated moment shows up in the $\mathrm{Cu}$-doped chromite. The reduced moment in $\mathrm{NiCr}_{2} \mathrm{O}_{4}$ indicates disorder on one $\mathrm{Cr}$ sublattice down to low temperature due to stronger frustration on the less distorted tetragonal pyrochlore lattice. Magnetoelastic effects and further neighbor spin interactions are mainly considered in the discussion of the observed peculiar ordering processes. Notable is the transition temperature of the $(1 / 2,1 / 2,1 / 2)$ phase of $\mathrm{NiCr}_{2} \mathrm{O}_{4}$ around $21 \mathrm{~K}$, where new anomalies for the specific heat and for the magnetodielectric behavior have recently been reported [T. D. Sparks et al., Phys. Rev. B 89, 024405 (2014)].

PACS number(s): 61.05.fm, 61.66.Fn, 71.70.Ej, 75.50.Gg 


\section{INTRODUCTION}

Compounds with antiferromagnetically (AF) coupled spins on the pyrochlore lattice are ideal systems to study the physics of geometrically frustrated magnets [1,2]. The pyrochlore network, due to its geometry of corner-sharing regular tetrahedra, has a very high degree of frustration; a simultaneous AF coupling on all six bonds of an individual tetrahedron becomes impossible. This results in a large degeneracy of the magnetic ground state and inhibits longrange order. On the other hand, short-range order will appear under the constraint of zero net moments on the tetrahedra.

In real systems weak perturbations like magnetoelastic coupling or further neighbor interactions will relieve frustration and establish long-range order. Therefore, the recent finding of a reduced moment on the orthorhombic distorted pyrochlore-type chromium lattice in the normal spinel $\mathrm{NiCr}_{2} \mathrm{O}_{4}$ [3] rises the general question how disorder, i.e. the absence of long range order, can remain and coexist with long-range order in the magnetic ground state. Normal spinels have the chemical composition $A B_{2} \mathrm{O}_{4}$. The $A^{2+}$ cation and $B^{3+}$ cation occupy the tetrahedral and octahedral sites, respectively, and the $B$ sites define the pyrochlore lattice. Many normal spinels with magnetic ions on both sites like in $\mathrm{NiCr}_{2} \mathrm{O}_{4}$ belong to the class of ferrimagnetic insulators and a few of them find applications as ferromagnetic materials. Essential to ferrimagnetism (FI) is a strong AF coupling between $A$ and $B$, so that the net moments of the two sublattices $A$ and $B$ align antiparallel. The presence of a significant AF coupling of $B-B$ direct neighbors can cause an additional AF phase by $B$ components lying in a plane perpendicular to the FI components resulting in a canted spin structure. This type of magnetism is reported for $\mathrm{NiCr}_{2} \mathrm{O}_{4}$ [3]. Important for the analysis of the magnetic order is a cooperative structural transition from cubic to tetragonal with an elongated tetragonal axis induced by Jahn-Teller active $\mathrm{Ni}^{2+}$ ions. A further lattice distortion from tetragonal to orthorhombic symmetry occurs around $70 \mathrm{~K}$ with the onset of FI ordering with a propagation vector $\boldsymbol{k}=(0,0,0)$ [3-5]. The AF order on the $\mathrm{Cr}$ ions occurs below $32 \mathrm{~K}[3,6]$. Controversial are two recently proposed models for the AF phase [3,6]. Our objective is to clarify the magnetic ground state of $\mathrm{NiCr}_{2} \mathrm{O}_{4}$ and relate the magnetic structure to possible magnetoelastic effects or further neighbor magnetic interactions. The influence of magnetoelastic effects becomes accessible if structural distortions can be tuned e.g. by chemical substitutions on the $A$ site of the spinel. Therefore, we also perform neutron diffraction on $\mathrm{Ni}_{0.98} \mathrm{Cu}_{0.02} \mathrm{Cr}_{2} \mathrm{O}_{4}$ to decrease the level of frustration. The Jahn-Teller active $\mathrm{Cu}^{2+}$ ion "flattens" the $\mathrm{Cr}_{4}$ 
tetrahedron, which differs from the elongation by the $\mathrm{Ni}^{2+}$ ion and allows to change the orthorhombic distortion [3].

Beside studies of magnetic properties the observation of magnetodielectricity in some spinels initiated research activities towards multiferroic properties of these materials as well. Recently, $\mathrm{NiCr}_{2} \mathrm{O}_{4}$ was identified as a magnetodielectric material [7], which is categorized as a type II multiferroic [8]. Magnetocapacitance reveals a magnetodielectric hysteresis and a new anomaly at $20 \mathrm{~K}$ that can relate to changes in the magnetic structure [5,7]. This anomaly is attributed to a spin driven structural change resulting from the completion of magnetic ordering [5]. Furthermore, an anomaly in the specific heat around this AF transition has a peculiar shape, suggesting two kinks separated by $2 \mathrm{~K}$, and could indicate two transitions [9]. Altogether, this points towards a more complicated magnetic behavior of the antiferromagnetic ordering on a distorted pyrochlore lattice with succeeding ordering transitions below $31 \mathrm{~K}$ unraveled so far.

In order to study subtle magnetic and structural changes as a function of temperature we apply neutron powder diffraction with high counting statistics and resolution to uncover possible new phases and to gain insight about static magnetic disorder even present at low temperature. Magnetization measurements supplement the work.

\section{EXPERIMENTAL DETAILS}

Powder samples of $\mathrm{NiCr}_{2} \mathrm{O}_{4}$ and $\mathrm{Ni}_{0.98} \mathrm{Cu}_{0.02} \mathrm{Cr}_{2} \mathrm{O}_{4}$ were prepared by the precursor method as described earlier [3]. The sample quality was checked by $x$-ray powder diffractometer (Bruker D8 advance) using $\mathrm{Cu}-\mathrm{K} \alpha$ radiation. The $x$-ray powder patterns of both samples only show minor amounts of $\mathrm{Cr}_{2} \mathrm{O}_{3}\left(3.5 \%\right.$ for $\mathrm{NiCr}_{2} \mathrm{O}_{4}$, and $0.4 \%$ for $\mathrm{Ni}_{0.98} \mathrm{Cu}_{0.02} \mathrm{Cr}_{2} \mathrm{O}_{4}$ ). Neutron powder diffraction experiments are carried out on the instruments E2, E6 and E9 at the BER II reactor of the Helmholtz-Zentrum Berlin (HZB) [10]. In order to investigate in detail changes of the crystal structure as a function of temperature complete powder patterns are collected on the high-resolution diffractometer E9 in the range $8^{\circ} \leq 2 \theta \leq 142^{\circ}$. This instrument uses a Ge monochromator selecting the neutron wavelengths $\lambda=1.3087$ or 1.7985 $\AA$. The magnetic ordering in $\mathrm{NiCr}_{2} \mathrm{O}_{4}$ and $\mathrm{Ni}_{0.98} \mathrm{Cu}_{0.02} \mathrm{Cr}_{2} \mathrm{O}_{4}$ is studied on the instruments E2 and E6, which both use a pyrolytic graphite monochromator selecting $\lambda=2.38$ and $2.42 \AA$, respectively. The instrument E2 is well suited to measure powder patterns with high resolution in low $q$ range, which allows us to separate magnetic reflections of coexisting 
magnetic phases. The instrument E6 is equipped with a double focusing monochromator yielding a high neutron flux. This allows us to follow in detail the thermal variation of the intensity of magnetic Bragg reflections and lattice parameters. Powder patterns are recorded between the diffraction angles from 15.7 to $91.3^{\circ}$ on E2 and from 7.5 to $136.5^{\circ}$ on E6. In addition, difference patterns from data sets collected at different temperatures are used to separate the reflections of particular magnetic structures. Rietveld refinements of the powder diffraction data are carried out with the program FullProf [11]. For the analysis of the neutron data we use the nuclear scattering lengths $b(\mathrm{O})=5.805 \mathrm{fm}, b(\mathrm{Cr})=3.635 \mathrm{fm}, b(\mathrm{Ni})=10.3$ $\mathrm{fm}$, and $b(\mathrm{Cu})=7.818 \mathrm{fm}$, respectively [12]. The magnetic form factors of the $\mathrm{Cr}^{3+}, \mathrm{Ni}^{2+}$ and

$\mathrm{Cu}^{2+}$ ions are taken from Ref. 13. Magnetization measurements as a function of temperature are carried out between 5 and $90 \mathrm{~K}$ using the vibrating sample magnetometer option of a Physical Properties Measurement System (PPMS, Quantum Design), which is part of the Quantum Materials Corelab at the HZB.

\section{RESULTS AND DATA ANALYSIS}

In order to investigate in detail the magnetic behavior of $\mathrm{NiCr}_{2} \mathrm{O}_{4}$ we have followed the temperature dependence of magnetic Bragg reflections in the range between 2 and $65 \mathrm{~K}$ where we find several magnetic phase transitions and coexistence of magnetic phases (Fig. 1). As recently reported the strongest magnetic intensity appears at the position of the reflection (1 111$)_{\mathrm{M}}$ due to the ferrimagnetic ordering of the $\mathrm{Ni}$ and $\mathrm{Cr}$ moments, which disappears above $T_{\mathrm{FI}}=74 \mathrm{~K}[3,6]$. At lower temperature the coupling between the $\mathrm{Cr}$ moments leads to an antiferromagnetic ordering, where the strongest commensurate magnetic reflections $\left(\begin{array}{lll}1 & 1 & 0\end{array}\right)_{\mathrm{M}}$ and $\left(\begin{array}{lll}2 & 0 & 1\end{array}\right)_{\mathrm{M}}$ are generated from the propagation vector $\boldsymbol{k}=(0,0,1)$. These reflections show a spontaneous change of their positions above $29 \mathrm{~K}$, where one finds an incommensurate (IC) phase with a vector $\boldsymbol{k}=(0,0,1-\delta)$ with $\delta=0.07(1)$ i.e. $k_{z}=0.93(1)$, and a possibly commensurate $(\mathrm{C})$ phase with the a vector $\boldsymbol{k}=(-\varepsilon, \varepsilon, 1)$ with $\varepsilon \cong 1 / 8$ (Fig. 1). Both magnetic phases are only stable in a small gap from $29 \mathrm{~K}$ up to the antiferromagnetic transition at $T_{\mathrm{AF}}=$ 31 K. Surprisingly, another new magnetic phase with the vector $\boldsymbol{k}=(1 / 2,1 / 2,1 / 2)$ is identified below $21 \mathrm{~K}$, which is present down to the lowest measured temperature of $1.7 \mathrm{~K}$. As shown in Fig. 1 the magnetic reflection $\left(-1 \frac{1 / 2}{1 / 2} 1 / 2\right)_{\mathrm{M}}$ is weak and hardly to observe, since it is close to the $2 \theta$ position of the strong magnetic reflection $\left(\begin{array}{lll}1 & 1 & 1\end{array}\right)_{\mathrm{M}}$. Despite this fact, our E2 measurement reveals a remarkable temperature-dependent behavior of the $\left(-1 \frac{1}{2} \quad \frac{1}{2} \quad \frac{1}{2}\right)_{M}$ 
intensity. It spontaneously appears below $21 \mathrm{~K}$, shows the maximum intensity from 19 to 15 $\mathrm{K}$, and strongly decreases down to $1.6 \mathrm{~K}$. Data sets between 15 and $2 \mathrm{~K}$ measured on E6 are not shown in Fig. 1. The reflections of the $(0,0,1) /\left(0,0, k_{z}\right)$ phase are not affected by the onset and the temperature dependent behavior of the $(1 / 2,1 / 2,1 / 2)$ phase.

A substitution of only $2 \%$ of $\mathrm{Ni}$ by $\mathrm{Cu}$ significantly changes the magnetic behavior of this system (Fig. 2). In contrast to $\mathrm{NiCr}_{2} \mathrm{O}_{4}$, the $(-\varepsilon, \varepsilon, 1)$ phase is not found in the patterns of $\mathrm{Ni}_{0.98} \mathrm{Cu}_{0.02} \mathrm{Cr}_{2} \mathrm{O}_{4}$. Similarily, the AF order sets in by an IC phase with the vector $\boldsymbol{k}=(0,0,1-\delta)$ with $\delta=0.09(1)$ i.e. $k_{z}=0.91(1)$ at $31 \mathrm{~K}$. Around $27 \mathrm{~K}$ one clearly observes the onset of the $(0,0,1)$ phase. The data do not allow to determine precisely the temperature for the onset of the latter phase. There is still the possibility that the $(0,0,0.91)$ phase is established together with weak contributions from the $(0,0,1)$ phase. However, according to Landau theory two magnetic vectors cannot become critical in a continuous transition at the same temperature. The magnetic Bragg intensities of the $(0,0,0.91)$ phase achieve a maximum at $27 \mathrm{~K}$, decrease towards $20 \mathrm{~K}$ and stay constant down to $2 \mathrm{~K}$. Fig. 3 shows the corresponding behavior for the refined moment. Tiny changes with temperature qualitatively similar to the behavior of the moment occur for the wave vector as well. We observe a continuous increase of intensities for the $(0,0,1)$ phase down to $20 \mathrm{~K}$. Thereafter the intensities become temperature independent down to $2 \mathrm{~K}$. The weak reflections of the $(1 / 2,1 / 2,1 / 2)$ phase, observed in $\mathrm{NiCr}_{2} \mathrm{O}_{4}$ below $21 \mathrm{~K}$, occur already below $24 \mathrm{~K}$ and are strongly intensified for $\mathrm{Ni}_{0.98} \mathrm{Cu}_{0.02} \mathrm{Cr}_{2} \mathrm{O}_{4}$ (Fig. 2).

First we analyze the magnetic structure measured at $1.6 \mathrm{~K}$, and particularly the $(0,0,1)$ phase. Fig. 4 shows four corner sharing $\mathrm{Cr}_{4}$ tetrahedra. The four spins on a $\mathrm{Cr}_{4}$ tetrahedron are denoted by $S 1$ to $S 4$. The Cr ions form chains along the two diagonals containing either $S 1-S 2$ (blue line) or $S 3-S 4$ (red line). The data of recent neutron diffraction measurements were analyzed using a model with an AF coupling $(\ldots+-+-+\ldots)$ for chains with $S 1-S 2$ and $S 3-$ $S 4$ [3]. This becomes likely due to the shortest $\mathrm{Cr}-\mathrm{Cr}$ distance for the orthorhombic distorted lattice [14]. The other four chains formed by $S 1-S 3, S 1-S 4, S 2-S 3$, and $S 2-S 4$ have an alternating $\mathrm{AF}$ and $\mathrm{F}$ bond, i.e. the spin sequences become $(\ldots++--++--++\ldots)$. The structure is collinear with a spin direction along $a_{3}$. The reported agreement between calculated and measured intensities is not satisfying. In particular, the intensity ratio of the 11 0 and 201 reflections has been measured to be about $20 \%$ below the calculated value for the structure above. This observation is in accordance with other experimental results [6]. It will be noted that a moment pointing along $a_{3}$ already maximizes this intensity ratio for the $(0,0,1)$ phase and it is still too low. 
A different collinear model, that is cited frequently, removes this discrepancy by proposing a $(0,0,1)$ structure with an AF bond for the pairs $S 1-S 3$ and $S 2-S 4$ [6]. The "saturated" AF component of the Cr moment for the two pairs become different. A small contribution of an $\mathrm{AF}$ bond for the $\mathrm{Ni}^{2+}$ ions was considered as well. That analysis was carried out using a pattern with a maximum diffraction angle slightly larger than those of the 220 and 202 reflections. In our measurements we find beyond this range the magnetic $\left(\begin{array}{lll}2 & 2 & 1\end{array}\right)_{\mathrm{M}}$ reflection to be absent. The structure factor $F(h k l)$ of a single $\mathrm{Cr}_{4}$ tetrahedron is calculated using the positions $\mathrm{Cr} 1(0,0,0), \mathrm{Cr} 2(1 / 4,1 / 4,0), \operatorname{Cr} 3(1 / 4,0,1 / 4), \mathrm{Cr} 4(0,1 / 4,1 / 4)$. For the reflection $(22$ 1) $)_{\mathrm{M}}$ the structure factor becomes

$$
F(221) \sim(S 1+S 2)+(S 3+S 4) e^{\frac{3}{2} \pi i} .
$$

This structure factor is zero only for $S 1=-S 2$ and $S 3=-S 4$. In Ref. 6 an AF-structure model is presented, where the Cr pairs $S 1-S 2$ and $S 3-S 4$ each have finite net moment along the $a_{3}$ direction. Therefore, that model contradicts the extinction rule above. This has additionally been crosschecked by a powder pattern simulation.

In our model we divide the $\mathrm{Cr}$ lattice into two sublattices according to the different chain directions in the $a_{1} a_{2}$ plane. That means the pair $S 1-S 2$ belongs to sublattice $I(S L-I)$, and $S 3$ $S 4$ to $S L-I I$. The extinction rule above states an AF bond for $S 1-S 2$ and $S 3-S 4$ allowing different moment sizes for each of the two pairs which has not been considered in [3]. Even more the diffraction patterns are identical for the AF-coupled spins as long as the value $\left(M_{S 1-S 2}\right)^{2}+\left(M_{S 3-S 4}\right)^{2}$ does not change. This can be seen from the structure factor for the $\mathrm{Cr}_{4}$ tetrahedron setting $S 2=-S 1$ and $S 4=-S 3$

$$
F(h k l) \sim S 1\left(1-e^{\frac{h+k}{4} 2 \pi i}\right)+S 3 e^{\frac{h+l+1}{4} 2 \pi i}\left(1-e^{\frac{k-h}{4} 2 \pi i}\right) .
$$

The values for $h+k$ and $k-h$ are even and the values within both brackets can only be 2 or zero. In addition, the $S 1$ term is real and the $S 3$ term is purely imaginary because $h+l+l$ is odd. Therefore the measured intensity $I \sim F^{2}=F_{\text {real }}^{2}+F^{2}$ im proves the statement above. We have used reflection conditions for space group Fddd. Structures with moments in SL-I and zero moments in the $S L-I I$ cannot be distinguished by powder diffraction from a structure with identical moments in both sublattices. For $\mathrm{NiCr}_{2} \mathrm{O}_{4}$ a moment of about $1.4 \mu_{\mathrm{B}}$ is found, assuming identical moments on both sublattices, and about $2 \mu_{\mathrm{B}}$ if the moments are located only in one sublattice.

We continue with the analysis of the $(1 / 2,1 / 2,1 / 2)$ phase. The powder pattern could not evidence magnetic intensities at the position of the reflection $\left(1 / 21 / 2 \frac{1}{2}\right)_{\mathrm{M}}$, but strong intensity for the reflection $\left(-1 \frac{1 / 2}{1 / 2} 1 / 2\right)_{M}$. For the reflection $(1 / 21 / 21 / 2)_{M}$ the structure factor becomes 


$$
F(1 / 2,1 / 2,1 / 2) \sim\left(S 1+S 2 e^{\frac{1}{2} \pi i}\right)+(S 3+S 4) e^{\frac{1}{2} \pi i} .
$$

An extinction of this reflection requests $S 1=S 2=0$ and $S 3=-S 4$ for a collinear structure. Therefore, AF-bonded Cr moments can only be on SL-II. In total four reflections $(1 / 21 / 21 / 2)$, $\left(1 / 2 \frac{1}{2},-1 / 2\right),(1 / 2-1 / 2,1 / 2)$, and $\left(-1 / 2{ }^{1 / 2}{ }^{1 / 2}\right)$ have to be considered. The two reflections $(1 / 21 / 2 \pm 1 / 2)$ request zero moments on $S L-I$ and AF bonding on $I I$. Reflections $\left(1 / 2-1 / 2 \frac{1}{2}\right)$ and $(-1 / 21 / 21 / 2)$ simply interchange $S L-I$ and $S L-I I$. Overall two domains become possible. Fits using this model with AF coupled moments located on SL-II result in a good agreement between calculated and measured patterns. The best fit is obtained for a moment orientation along $a_{3}$ (Table I). The observed peaks are broader than the instrumental resolution. A correlation length $L$ around $1 \cdot 10^{2} \AA$ is estimated from a peak broadening $\Delta(2 \theta)$ of $1 \cdot 10^{-2} \mathrm{rad}$ using $L=$ $\lambda / \cos \theta \Delta(2 \theta)$, where $\lambda$ is the neutron wavelength [15]. The moments determined at $1.6 \mathrm{~K}$ are about $0.5 \mu_{\mathrm{B}}$ for $\mathrm{NiCr}_{2} \mathrm{O}_{4}$ and $1.7 \mu_{\mathrm{B}}$ for $\mathrm{Ni}_{0.98} \mathrm{Cu}_{0.02} \mathrm{Cr}_{2} \mathrm{O}_{4}$. The results of the Rietveld refinement of $\mathrm{Ni}_{0.98} \mathrm{Cu}_{0.02} \mathrm{Cr}_{2} \mathrm{O}_{4}$ are shown in Fig. 5 and Tables II and III. It has to be noted that the reflections of the refined $(0,0,1-\delta)$ phase strongly overlap with reflections of the other magnetic phases of $\mathrm{Ni}_{0.98} \mathrm{Cu}_{0.02} \mathrm{Cr}_{2} \mathrm{O}_{4}$ as well as the magnetic $\left(\begin{array}{lll}0 & 1 & 2\end{array}\right)_{\mathrm{M}}$ reflection at $38.4^{\circ}$ of the impurity phase $\mathrm{Cr}_{2} \mathrm{O}_{3}$.

Diffuse scattering with a lorentzian like peak shape occurs above and below the FI transition. At about $36 \mathrm{~K}$, where the FI structure approaches saturation, the diffuse scattering has changed from lorentzian to a "deformed trapezoidal" shape in a limited $q$ range approximately located between the 1110 and 1112 reflections. This diffuse scattering decreases step like at each magnetic transition temperature as visible in the lower part of Fig. 2 for $\mathrm{Ni}_{0.98} \mathrm{Cu}_{0.02} \mathrm{Cr}_{2} \mathrm{O}_{4}$. The trapezoidal line shape mainly persists and the diffuse scattering transfers to magnetic Bragg scattering in the low temperature range with AF order.

Complementarily we have investigated the temperature dependent magnetization of $\mathrm{NiCr}_{2} \mathrm{O}_{4}$ and $\mathrm{Ni}_{.98} \mathrm{Cu}_{.02} \mathrm{Cr}_{2} \mathrm{O}_{4}$ in the range from 4 to $90 \mathrm{~K}$ in a $d c$ field of $0.05 \mathrm{~T}$ presented in Fig. 6. Both chromites show a spontaneous increase of magnetization at the Curie temperature $T_{\mathrm{C}}=66 \mathrm{~K}$. In the magnetically ordered range two anomalies appear for $\mathrm{Ni}_{0.98} \mathrm{Cu}_{0.02} \mathrm{Cr}_{2} \mathrm{O}_{4}$ at the magnetic transition temperatures 31 and $23 \mathrm{~K}$, which indicate the onset of antiferromagnetic order of the coexisting $(0,0,1)$ and $(1 / 2,1 / 2,1 / 2)$ phases. In contrast, $\mathrm{NiCr}_{2} \mathrm{O}_{4}$ has only one anomaly at the AF transition at $32 \mathrm{~K}$, in agreement with earlier magnetization measurements $[6,16]$. The ordered moment of about $0.5 \mu_{\mathrm{B}}$ for the $(1 / 2,1 / 2,1 / 2)$ transition probably weakens the anomaly so that it becomes nonvisible in the measurements. The saturated magnetization value of $\mathrm{Ni}_{0.98} \mathrm{Cu}_{0.02} \mathrm{Cr}_{2} \mathrm{O}_{4}$ at $5 \mathrm{~K}$ is found to be slightly smaller than that of $\mathrm{NiCr}_{2} \mathrm{O}_{4}$. 
In the magnetically ordered state, $\mathrm{NiCr}_{2} \mathrm{O}_{4}$ and $\mathrm{Ni}_{0.98} \mathrm{Cu}_{0.02} \mathrm{Cr}_{2} \mathrm{O}_{4}$ exhibit orthorhombic distortions leading to a crystal structure with the space group $F d d d$. Rietveld refinements have been carried out to determine the lattice parameters labelled as $a_{i}$ with $a_{1}<a_{2}<a_{3}$, which are summarized in Table II. For $\mathrm{NiCr}_{2} \mathrm{O}_{4}$ the lattice parameters as well as the distortions within the planes, derived from dist $t_{i j}=2\left(a_{i}-a_{j}\right) /\left(a_{i}+a_{j}\right)$, only show tiny temperature dependences. On the other hand, the temperature behavior of the lattice parameters can be strongly influenced by a replacement of only $2 \% \mathrm{Ni}$ by $\mathrm{Cu}$. In Table II a significant distortion from dist $_{21}=2.3$ to 3.5 within the $a_{1} a_{2}$ plane can be seen from 4 to $25 \mathrm{~K}$, respectively. This distortion is related to a decrease of $a_{1}$ and a five times larger increase of $a_{2}$. In order to follow the change of the lattice distortions in $\mathrm{Ni}_{0.98} \mathrm{Cu}_{0.02} \mathrm{Cr}_{2} \mathrm{O}_{4}$ as a function of temperature we have measured the strong peak observed at $102.2^{\circ}$ between 19 and $27 \mathrm{~K}$. This peak mainly contains intensity of the overlapping reflections 484 and 844 . In Fig. 8 it can be seen that this peak shows a spontaneous broadening below $24 \mathrm{~K}$. Close to this temperature we find the onset of the second antiferromagnetic transition at around $24 \mathrm{~K}$ determined from neutron diffraction and around $23 \mathrm{~K}$ from the magnetization measurements, respectively. This peak broadening can be ascribed to the shifts of the 484 and 844 reflections to higher and lower diffraction angles, respectively (Fig. 8).

\section{DISCUSSION AND CONCLUSION}

Lattice distortions generated by spin-lattice coupling in frustrated normal spinels $A B_{2} \mathrm{O}_{4}$ with nonmagnetic $A^{2+}$ ions can initiate a symmetry reduction lifting frustration and creating

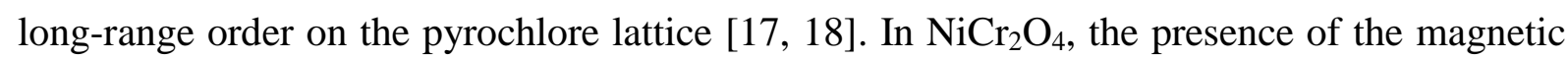
$\mathrm{Ni}^{2+}$ ion on the $A$ site already induces a distortion from cubic to tetragonal by the Jahn-Teller effect, and a further reduction to an orthorhombic symmetry sets in concomitantly with ferrimagnetic ordering [4-6]. The lattice distortion in the ferrimagnetic state restricts the number of possible degenerate AF structures for the Cr-ions on the pyrochlore lattice. The magnetic Ni-Cr and $\mathrm{Cr}-\mathrm{Cr}$ exchanges mainly cause canted structures with coexisting FI and AF magnetism and can confine the spatial dimensionality for the AF spin orientations. Possible magnetic structures as a function of the relative strengths for the three magnetic exchanges $J_{B B}, J_{A B}$ and $J_{A A}$ have been calculated for spinels in general, and tetragonal spinels in particular $[19,20]$. 
Both studied systems have a strong tetragonal, but a weak orthorhombic distortion that is about $40 \%$ larger in $\mathrm{Ni}_{0.98} \mathrm{Cu}_{0.02} \mathrm{Cr}_{2} \mathrm{O}_{4}$ compared to $\mathrm{NiCr}_{2} \mathrm{O}_{4}$ (Table II). As already pointed out the shortest Cr-Cr distance for the spin pairs $S 1-S 2$ and $S 3-S 4$ generate two strong bonds and four weak bonds of comparable strength on the $\mathrm{Cr}_{4}$ tetrahedron. The frustration index, gauging the degree of frustration and defined by the ratio of the Curie-Weiss and the Néel temperature is 7.8 [4]. An opposite situation occurs in $\mathrm{CuCr}_{2} \mathrm{O}_{4}$, where the Jahn-Teller active $\mathrm{Cu}^{2+}$ ion flattens the $\mathrm{CuO}_{4}$ tetrahedron and generates four strong and two weak bonds on the $\mathrm{Cr}_{4}$ tetrahedron. Antiferromagnetic coupling should establish on the strong bonds and the spin pairs of the weak bonds should be forced to a parallel alignment which is in agreement with the outcome of neutron diffraction reporting a moment of about $2 \mu_{\mathrm{B}}$ for the AF component of $\mathrm{Cr}$ [3]. The degree of frustration is quite low with an index of 1.1 [4].

Next we introduce some single-ion properties of the magnetic $\mathrm{Ni}^{2+}\left(\mathrm{Cu}^{2+}\right)$ and the $\mathrm{Cr}^{3+}$ ions and consider the local coordination formed by the nearest oxygen neighbors. All Ni ions reside on the special position $8 a$ of the space group $F d d d$. The ordered magnetic moment in the ground state (Table III) differs not much from the spin-only value of $2 \mu_{\mathrm{B}}$ for $\mathrm{Ni}^{2+}$ with $d^{8}$ configuration in a high-spin state. The canted $\mathrm{Cr}$ moments, located at the special position $16 d$, have their small component along the shortest lattice constant forming together with $\mathrm{Ni}$ ions the ferrimagnetic order. The large AF component adjusts along the longest lattice constant. A value around $3 \mu_{\mathrm{B}}$ for the total moment calculated for the $\mathrm{Cr}^{3+}$ ions on $S L I$ (Table III) agrees with a high-spin state for the electronic $d^{3}$ configuration. All ions $\mathrm{Ni}^{2+}$ and $\mathrm{Cu}^{2+}$ are located on the tetrahedral site. Local tetragonal distortions of opposite sign are induced by the JahnTeller effect for $\mathrm{Ni}$ and $\mathrm{Cu}$, respectively. In an earlier study of Kataoka and Kanamori a detailed description of the mixed $\mathrm{Ni}$ and $\mathrm{Cu}$ chromite is given considering the different types of local distortions and the observed bulk tetragonal distortion [21]. The essential point is that the long-lattice axis of the elongated $\mathrm{Ni}$ tetrahedron points along one of the two long axes of the flattened $\mathrm{Cu}$ tetrahedron. This will have an influence on the magnetic anisotropy, since the $\mathrm{Ni}^{2+}$ ions in majority, taking into account the anisotropy strength as well, can control the orientation of the JT distortion for the $\mathrm{Cu}^{2+}$ ions in minority. In our case with only $2 \%$ doping by $\mathrm{Cu}^{2+}$, single-ion anisotropy is considered only for $\mathrm{Ni}$ and $\mathrm{Cr}$. The observed magnetic anisotropy can originate from the single-site symmetry of the local crystal field, and the spinorbit coupling becomes the driving mechanism to couple spin moments to the lattice [22]. The anisotropy part of the Hamiltonian is described by $H=-\sum A_{i} S_{i}^{2}$, where the magnitude of $A_{i}$ determines the moment orientation along different directions [23]. In the case $A_{i} S_{i}^{2}>A_{j} S_{j}^{2}$, where $i$ indicates the long and $j$ one of the short lattice constants, the long lattice constant 
becomes the easy axis of magnetization. Then the ferrimagnetic moments align along the direction of short lattice constant axis as determined by the single-ion anisotropy of $\mathrm{Ni}$.

We now discuss common features of the magnetic ordering on sublattice $I$ for the pure and $\mathrm{Cu}$-doped sample. Both systems have two superposed structures with comparable moments for the $(0,0,1)$ and $\left(0,0, k_{z}\right)$ structures, respectively (Table III). This indicates that a doping of $2 \% \mathrm{Cu}$ is of low relevance for the magnetic order at low temperature. It is only the presence of the $(-\varepsilon, \varepsilon, 1)$ phase around the AF transition, which is present in the pure sample but absent in the doped one. The onset of antiferromagnetic order is continuous and dominated by the incommensurate sinusoidal phase (Fig. 3). The Fourier component of the moment for the IC phase has a maximum around $27 \mathrm{~K}$, decreases gradually and remains stable below $21 \mathrm{~K}$. On the other hand the Fourier component of the $\mathrm{C}$ phase slowly increases below $31 \mathrm{~K}$ and becomes saturated at about $22 \mathrm{~K}$. The sum of both Fourier components shows qualitatively the typical temperature dependence of an order parameter for a second order phase transition. Below $27 \mathrm{~K}$ this "order parameter" approaches saturation. However, the magnitude of the ordered moment varies on different sites due to the sinusoidal modulation for $\left(0,0, k_{z}\right)$, while the moment is saturated for $(0,0,1)$ on all sites. Free energy could then be gained by a transfer of the "Fourier component" from $\left(0,0, k_{z}\right)$ to $(0,0,1)$. Below $22 \mathrm{~K}$ no further transfer is observed resulting in a state, where the $(0,0,1)$ phase is now dominating, however, the $\left(0,0, k_{z}\right)$ structure is still present. Close to this temperature one finds the onset of the $(1 / 2,1 / 2,1 / 2)$ structure on SL II, which is probably described by a second order transition. Although a direct classification of the $(1 / 2,1 / 2,1 / 2)$ transition from our neutron data is not possible, since moments become too weak. On the other hand the magnetization measurements of the $\mathrm{Cu}$-doped sample show a continuous anomaly favoring a second order transition, which is similar to the AF transition (Fig. 6). The $(1 / 2,1 / 2,1 / 2)$ phase also occurs in the pure Ni chromite, but with strongly reduced moment given in Table III.

The analyzed collinearity of all spins in AF phases becomes plausible because the FI phase confines the moments for the AF phases to a perpendicular plane. This restricts spin fluctuations to two dimensions and thus differs from spinels with nonmagnetic $A$ ion, where, in the absence of anisotropy, fluctuations are possible along all three spatial directions. Fluctuations limited to two dimensions favor collinear magnetic structures due to "order from disorder" [24]. Concluding, collinear AF structures can become likely.

Synchrotron diffraction at $\mathrm{NiCr}_{2} \mathrm{O}_{4}$ showed a tiny anomaly in the lattice constant $a_{2}$ at 20 $\mathrm{K}$ indicating a small reduction of the orthorhombic distortion [4,5]. Moreover, magnetodielectric measurements revealed a new transition at $20 \mathrm{~K}$, which is attributed to the 
completion of magnetic order [5]. Our neutron data do not allow to measure such small lattice distortions, however, around $21 \mathrm{~K}$, we identify a magnetic transition to the $(1 / 2,1 / 2,1 / 2)$ phase correlating with the lattice and the dielectric anomaly. A qualitatively similar strongly enhanced lattice distortion becomes visible for $\mathrm{Ni}_{0.98} \mathrm{Cu}_{0.02} \mathrm{Cr}_{2} \mathrm{O}_{4}$ in our high-resolution neutron data for overlapping Bragg reflections $h k l$ and $k h l$ with largely different values for $h$ and $k$. Changes in peak width reflect the reduction of the orthorhombic distortion (Fig. 7), which is quantified by the outcome of the refinements (Table II). The larger moment of $1.7 \mu_{\mathrm{B}}$ for the $(1 / 2,1 / 2,1 / 2)$ phase increases the spin-lattice coupling in comparison to $\mathrm{NiCr}_{2} \mathrm{O}_{4}$ which has only a moment of $0.5 \mu_{\mathrm{B}}$. Surprisingly, no lattice distortion is visible in our data at the 2-k transition $(0,0,1)$ and $(0,0,0.91)$.

The slight decrease in magnetization with increasing $\mathrm{Cu}$ content between the pure Nichromite and the $\mathrm{Cu}$-doped chromite reflects the lowering of the moment on the $A$-ion site (Fig. 6). If the average moment generated at the $A$ sites outbalances the contribution from the $\mathrm{Cr}$-sites a reduction in the A-moment reduces the magnetization. The two anomalies in the temperature dependent magnetization around $31 \mathrm{~K}$ and $23 \mathrm{~K}$ for $\mathrm{Ni}_{0.98} \mathrm{Cu}_{0.02} \mathrm{Cr}_{2} \mathrm{O}_{4}$ (Fig. 6) relate very well to the magnetic phase transitions found by neutron diffraction. Both anomalies are attributed to a different canting of the Cr moments. At each transition " $(0,0,1)$ " and " $(1 / 2,1 / 2,1 / 2)$ " the onset of a mean field by AF ordered $\mathrm{Cr}$ ions exerts a torque on the total $\mathrm{Cr}$ moment and canting increases. That reduces the $\mathrm{Cr}$ moment along $a_{1}$, thus enlarges the difference between $\mathrm{Ni} / \mathrm{Cu}$ and $\mathrm{Cr}$ moment, and results in the observed increased magnetization. The magnetization measurement strongly support the presence of a larger magnetization on the $A$ sublattice with respect to the $B$ sublattice. The absence of the second anomaly for $\mathrm{NiCr}_{2} \mathrm{O}_{4}$ is attributed to the weakness of the $(1 / 2,1 / 2,1 / 2)$ phase.

Next, we compare our findings with magnetic structure calculations on a pyrochlore lattice considering tetragonal and orthorhombic distortions generated by spin-lattice coupling $[17,18]$. These calculations are based on nearest neighbor-exchange between $\mathrm{Cr}$ ions. In particular the observed $(0,0,1)$ structure is related to the lattice distortion described by a $q=0$ phonon of $E_{u}$ symmetry where two types of tetrahedra occur with perpendicular orientation of the orthorhombic distortion (Fig. 6 in [18] and Fig. 8). Each tetrahedron is surrounded by four tetrahedra with a perpendicular distortion. The double- $k$ structure $(1 / 2,1 / 2,1 / 2) /(0,0,1)$ also possesses these two types of distortions, however, each tetrahedron is surrounded by one of the same type of distortion and three with perpendicular distortion. We denote the two different orientations in the distortions by + and - . Then the distortions for $(0,0,1)$ have an alternating +-+- sequence along both diagonals, while the sequence for the double-k 
structure becomes +-+- along one and ++--++ along the other diagonal (Fig. 8). Always four tetrahedra of each type are located within the unit cell, thus averaging to a tetragonal geometry. Mainly we point out from this analysis that the internal local strain distribution is different between the double- $\boldsymbol{k}$ structure $(1 / 2,1 / 2,1 / 2) /(0,0,1)$ and the single- $\boldsymbol{k}$ structure $(0,0,1)$.

We now consider further neighbor interactions that can perturb frustration (Fig. 4). The strengths of these interactions labelled $J_{\mathrm{i}}$ with the index specifying the shells for neighbors, have been calculated for chromites with nonmagnetic $A$ ions [25]. We group the different interactions, up to third neighbor shell, into intrachain couplings, interchain couplings with spins on the same sublattice, and interchain couplings between spins belonging to different sublattices.

- Intrachain interactions are given by $J_{1}$ coupling $S 1-S 2$ and $J_{3}$ ' coupling $S 1-S 1$ '. The dominant exchange $J_{1}$ generates the strong AF-bonds on the chains in the $a_{1} a_{2}$ plane. The third nearest neighbor exchange $J_{3}$ ' couples second neighbor spins on the same chain. Chains in the SL-I and SL-II are identical and have the same magnetic energy.

- Interchain interactions between spins on the same sublattice are controlled by $J_{3}$,' coupling $S 1-S 1$ '. An AF $J_{3}$ "' bond for the determined $(0,0,1)$ phase is always present between chains inside the $a_{1} a_{2}$ plane. This differs for the $(1 / 2,1 / 2,1 / 2)$ phase where both $\mathrm{AF}$ and $\mathrm{F}$ bonds are present.

- The interchain interaction between the two $S L-I$ and $S L-I I$ described by $J_{2}$ has to be very small. On one hand, the size of $J_{2}$ was calculated to be small [25]. On the other hand, a spin on $S L-I$ like $S 1$ couples to a pair of oppositely aligned spins like S3' and S4', both residing on a chain of SL-II. This is shown by the two broken lines for $S 1-S 3$ ' and $S 1-S 4$ (Fig. 4).

The considerations above indicate that the interactions $J_{1}$ and $J_{3}$ ' determine the spin order on the linear chains. Assuming $J_{1}$ to be AF, competing interactions have to be considered for the case of an AF-type $J_{3}$ ' coupling and modulated structures become possible. If the sublattices are effectively decoupled due to a very weak $J_{2}$, the presence of a weak $J_{3}$ "' can drive three dimensional (3-d) order within one sublattice. Since the $J_{3}$ ''-bonds differ for both sublattices, differing order temperatures are expected. We observe a higher ordering temperature for the $(0,0,1)$ than for the $(1 / 2,1 / 2,1 / 2)$ phase that could favor an AF-coupling by $J_{3}$ "' . However, the AF 
and $\mathrm{F}$ bonds for the $(1 / 2,1 / 2,1 / 2)$ phase will approximately compensate and will not support an onset of 3 -d order. The observation that the order temperature for the $(1 / 2,1 / 2,1 / 2)$ phase becomes lower with decreasing orthorhombic distortion gives evidence for spin-lattice coupling.

In summary we find for $\mathrm{NiCr}_{2} \mathrm{O}_{4}$ a division of the magnetic $\mathrm{Cr}$ lattice into two sublattices. The spin moments on one sublattice become nearly saturated at low temperature. The moments on the other sublattice are strongly suppressed. The sublattice structures are characterized by different magnetic propagation vectors. Already a $2 \%$ substitution of Ni by $\mathrm{Cu}$ in pure Ni chromite is sufficient to restore almost the full moment on the second sublattice while maintaining the spin structure. It seems that the addition of $\mathrm{Cu}$, associated with a stronger orthorhombic distortion, influences the strength of the magnetic bonds. Remarkable is the absence and presence of magnetoelastic coupling for $\mathrm{Ni}_{0.98} \mathrm{Cu}_{0.02} \mathrm{Cr}_{2} \mathrm{O}_{4}$ at the order transitions for $S L-I$ around $31 \mathrm{~K}$ and $S L-I I$ around $23 \mathrm{~K}$, respectively. In both cases magnetic order occurs along only one of the diagonals. An increase of the orthogonal lattice distortion, however, occurs only around $23 \mathrm{~K}$ and not around $31 \mathrm{~K}$ [Table II]. This distortion can only be generated if magnetic order is present along both diagonal chains.

Finally, we compare our results with the magnetism in other frustrated systems, where different magnetic states occur with coexisting long-range order and disorder, usually shortrange order. Intensively studied are systems with cubic pyrochlore and kagome lattices. The cubic pyrochlore lattice, realized in some spinels, can be considered as an alternating stacking of a kagome and a triangular lattice. A neutron-scattering study of the spinel $\mathrm{LiMn}_{2} \mathrm{O}_{4}$ shows long- and short-range order with a correlation length associated with the magnetic nearest neighbor coupling [26]. The diffuse scattering is attributed to a partial spin-glass state, and a context to partial charge order of $\mathrm{Mn}^{3+} / \mathrm{Mn}^{4+}$ is considered. In our case, the pure Ni chromite is stoichiometric and spin-glass behavior is at least not associated with disorder on the lattice. A fully ordered state of canted spins on the kagome lattice and a completely disorder state on the triangular lattice is reported for the pyrochlore material $\mathrm{Gd}_{2} \mathrm{Ti}_{2} \mathrm{O}_{7}$ [27]. Here a 3/1 ratio for ordered/disordered spins is realized. Recently a 2/1 ratio was found for $\mathrm{Fe}_{4} \mathrm{Si}_{2} \mathrm{Sn}_{7} \mathrm{O}_{16}$ showing a stripe-like pattern with ordered AF and canted spins on chains separated by disordered interstitial spins between the chains [28]. No magnetostrictive lattice changes were observed despite the fact that the hexagonal symmetry is broken. Similarily for the Ni chromite we do not observe magnetostrictive lattice distortions at the transition into the $(0,0,1) /\left(0,0, k_{z}\right)$ phase. In contrast, lattice distortions appear at the transition into the $(1 / 2,1 / 2,1 / 2)$ phase. Another state of coexisting order and disorder on a kagome lattice is established in $\mathrm{Dy}_{3} \mathrm{Mg}_{2} \mathrm{Sb}_{3} \mathrm{O}_{14}$ [29]. Evidence is found for a transition into an emergent charge order, where different microstates 
are present. Averaging over these states lead to a strong reduction of the spin moments by roughly one third, and this specimen is a nice example for exotic states in frustrated systems.

In conclusion a thorough understanding of order/disorder and the observed sublattice formation has at least to take into account magnetoelastic effects and the contribution to elastic energy from orthorhombic or other lattice distortions. The recent observation of magnetodielectric behavior at a phase transition temperature around $21 \mathrm{~K}$ [5] combined with our finding of a magnetic sublattice order at the same temperature points towards a complicated interplay of magnetic, dielectric, and elastic properties for $\mathrm{NiCr}_{2} \mathrm{O}_{4}$.

\section{Acknowledgement}

The authors thank T. Hofmann for critical reading of the manuscript.

\section{References}

[1] J. T. Chalker, Geometrically Frustrated Antiferromagnets: Statistical Mechanics and Dynamics, in Introduction to Frustrated Magnetism, edited by C. Lacroix, P. Mendels, and F. Mila, Springer Series in Solid State Science 164, 3 (2011).

[2] L. Balents, Nature 464, 207 (2010).

[3] M. Reehuis, M. Tovar, D. M. Többens, P. Pattison, A. Hoser, and B. Lake, Phys. Rev. B 91, 024407 (2015).

[4] M. R. Suchomel, D. P. Shoemaker, L. Ribaud, M. C. Kemei, and R. Seshadri, Phys. Rev. B 86, 054406 (2012).

[5] T. D. Sparks, M. C. Kemei, P. T. Barton, and R. Seshadri, Phys. Rev. B 89, 024405(2014).

[6] K. Tomiyasu and I. Kagomiya, J. Phys. Soc. Jpn. 73, 2539 (2004).

[7] N. Mufti, A. A. Nugroho, G. R. Blake, and T. T. M. Palstra, J. Phys.: Condens. Matter 22, 075902 (2010).

[8] A. Maignan, C. Martin, K. Singh, Ch. Simon, O. I. Lebedev, and S. Turner, J. Solid State Chem. 195, 41 (2012).

[9] S. Klemme and J. C. van Miltenburg, Phys. Chem. Minerals 29, 663 (2002).

[10] https://www.helmholtz-berlin.de/media/media/nutzerdienst/experimental_facilities

[11] J. Rodríguez-Carvajal, Physica B 192, 55 (1993). 
[12] V. F. Sears, in International Tables for Crystallography, edited by Wilson, A. J. C. (Kluwer Academic Publishers, Dordrecht/Boston/London, 1995), C, 383.

[13] P. J. Brown, in International Tables for Crystallography, edited by A. J. C. Wilson (Kluwer Academic Publishers, Dordrecht/Boston/London, 1995), C, 391.

[14] K. Motida and S. Miyahara, J. Phys. Soc. Jpn. 28, 1188 (1970).

[15] A. Guinier, X-Ray Diffraction in Crystals, Imperfect Crystals and Amorphous Bodies (New York, Dover, 1994).

[16] H. Ishibashi and T. Yasumi, J. Magn. Magn. Mater. 310, e610 (2007).

[17] O. Tchernyshyov, R. Moessner, and S. L. Sondhi, Phys. Rev. Lett. 88, 067203 (2002).

[18] O. Tchernyshyov, R. Moessner, and S. L. Sondhi, Phys. Rev. B 66, 064403 (2002).

[19] T. A. Kaplan and N. Menyuk, Phil. Mag. 87, 3711 (2007).

[20] N. Menyuk, K. Dwight, D. Lyons, and T. A. Kaplan, Phys. Rev. 127, 1983 (1962).

[21] M. Katoka and J. Kanamori, J. Phys. Soc. Jpn. 32, 113 (1972).

[22] G. F. Dionne, Magnetic Oxides (Springer, New York/Dordrecht/Heidelberg/London, 2009).

[23] S. Maekawa, T. Tohyama, S. E. Barnes, S. Ishihara, W. Koshibae, G. Khaliullin, Physics of Transition Metal Oxides, Springer Series in Solid State Science 144, 1 (Springer, Berlin Heidelberg/ New York, 2004).

[24] R. Moessner and J. T. Chalker, Phys. Rev. B 5812049 (1998).

[25] A. N. Yaresko, Phys. Rev. B 77, 115106 (2008).

[26] J. E. Greedan, C. R. Wiebe, A. S. Wills, J. R. Steward, Phys. Rev B 65, 184424 (2002).

[27] J. D. M. Champion, A. S. Wills, T. Fennel, S. T. Bramwell, J. S. Gardner, and M. A. Green, Phys. Rev B 64, 140407(2001).

[28] C. D. Ling, M. C. Allison, S. Schmid, M. Avdeev, J. S. Gardner, C.-W. Wang, D. H. Ryan, M. Zbiri, and T. Söhnel, Phys. Rev. B 96, 180410(R) (2017).

[29] J. A. M. Paddison, H. S. Ong, J. O. Hamp, P. Mukherjee. X. Bai, M. G. Tucker, N. P. Butch, C. Castelnovo, M. Mourigal, and S. E. Dutton, Nature Comm. 7, 13842 (2016). 
TABLE I. Observed peak intensities for $(1 / 2,1 / 2,1 / 2)$ phase of $\mathrm{Ni}_{0.98} \mathrm{Cu}_{0.02} \mathrm{Cr}_{2} \mathrm{O}_{4}$ and calculated intensities for collinear structure with moments either along $y$ or along $z$ direction. The intensities are the sum of $h k l$ and $k h l$ reflections e.g. $I\left(-1 \frac{1 / 2}{1 / 2}{ }^{1 / 2}\right)=I\left(-1 \frac{1}{2} 1^{1 / 2} 1 / 2\right)+I\left(1 / 2-1 \frac{1}{2}\right.$ $1 / 2)$. Given numbers are intensities normalized to 1000 for $I\left(1 \frac{1}{2}-1 / 21 / 2\right)$. Standard deviations of the measured intensities amount to about $5 \%$.

\begin{tabular}{lllll}
\hline \hline \multicolumn{1}{c}{$h k l$} & $-1 \frac{1 / 2^{1 / 2} 2^{1 / 2}}{1 \frac{1}{2} 2^{1 / 2} 1 \frac{1}{1 / 2} 2^{1 / 2}}$ & 111 \\
\hline Diffraction angle & $27.9^{\circ}$ & $36.3^{\circ}$ & $44.5^{\circ}$ & $28.6^{\circ}$ \\
$F W H M$ & $0.93(4)^{\circ}$ & $0.66(5)^{\circ}$ & $0.85(3)^{\circ}$ & $0.48(1)^{\circ}$ \\
Integr. obs. Int. & 1000 & 360 & 344 & 1000 \\
Cal. Int. $(m / / z)$ & 1000 & 311 & 311 & \\
Cal. Int. $(m / / y)$ & 1000 & 693 & 283 & \\
\hline \hline
\end{tabular}

TABLE II. Results of the Rietveld refinements of $\mathrm{NiCr}_{2} \mathrm{O}_{4}$ and $\mathrm{Ni}_{0.98} \mathrm{Cu}_{0.02} \mathrm{Cr}_{2} \mathrm{O}_{4}$ from neutron powder data collected on the instrument E9. The lattice parameters $a_{i}$ and the three positional parameters of the oxygen were determined for both chromites at several temperatures from refinements using the space group $F d d d$. The calculated cell distortion ns $d i s t_{i j}=2\left(a_{i}-a_{j}\right) /\left(a_{i}\right.$ $+a_{j}$ ) and the cell volumes are also listed. The given residual $R_{F}$ of each refinement is defined as $\left.R_{F}=\sum|| F_{\text {obs }}|-| F_{\text {calc }}|| / \sum\left|F_{\text {obs }}\right|\right)$.

\begin{tabular}{|c|c|c|c|c|c|c|c|c|}
\hline$x(\mathrm{Cu})$ & 0.00 & 0.00 & 0.00 & 0.00 & 0.00 & 0.02 & 0.02 & 0.02 \\
\hline$T[\mathrm{~K}]$ & 2 & 20 & 25 & 30 & 35 & 4 & 25 & 32 \\
\hline$x(\mathrm{O})$ & $0.2560(4)$ & $0.2556(4)$ & $0.2563(4)$ & $0.2558(4)$ & $0.2559(4)$ & $0.2567(5)$ & $0.2562(3)$ & $0.2577(3)$ \\
\hline$y(\mathrm{O})$ & $0.2599(4)$ & $0.2600(4)$ & $0.2594(4)$ & $0.2601(4)$ & $0.2601(4)$ & $0.2591(5)$ & $0.2595(3)$ & $0.2582(3)$ \\
\hline$z(\mathrm{O})$ & $0.2679(1)$ & $0.2680(2)$ & $0.2679(1)$ & $0.2679(1)$ & $0.2678(1)$ & $0.2687(2)$ & $0.2685(2)$ & $0.2686(2)$ \\
\hline$a_{1}[\AA ̊ \AA 0]$ & $8.1640(3)$ & $8.1640(3)$ & $8.1646(3)$ & $8.1660(2)$ & $8.1674(3)$ & $8.1725(5)$ & $8.1708(4)$ & $8.1728(4)$ \\
\hline$a_{2}[\AA]$ & $8.1773(3)$ & $8.1770(3)$ & $8.1786(3)$ & $8.1798(3)$ & $8.1806(3)$ & $8.1910(5)$ & $8.1992(4)$ & $8.2003(4)$ \\
\hline$a_{3}[\AA]$ & $8.5673(2)$ & $8.5663(2)$ & $8.5661(2)$ & $8.5643(2)$ & $8.5626(2)$ & $8.5781(3)$ & $8.5784(3)$ & $8.5757(4)$ \\
\hline $\operatorname{dist}_{21}\left[10^{-3}\right]$ & 1.6 & 1.6 & 1.7 & 1.7 & 1.6 & 2.3 & 3.5 & 3.4 \\
\hline $\operatorname{dist}_{31}\left[10^{-3}\right]$ & 48.2 & 48.1 & 48.0 & 47.6 & 47.2 & 48.4 & 48.7 & 48.1 \\
\hline $\operatorname{dist}_{32}\left[10^{-3}\right]$ & 46.6 & 46.5 & 46.3 & 45.9 & 45.6 & 46.2 & 45.2 & 44.8 \\
\hline$V\left[\AA^{3}\right]$ & $571.95(3)$ & $571.86(3)$ & $572.00(3)$ & $572.06(3)$ & $572.10(3)$ & $574.22(3)$ & $574.70(3)$ & $574.74(3)$ \\
\hline$R_{F}$ & 0.0245 & 0.0233 & 0.0220 & 0.0255 & 0.0233 & 0.0318 & 0.0293 & 0.0280 \\
\hline
\end{tabular}


TABLE III. Fourier component of moments for the ferri- (FI) and antiferromagnetic (AF) phases obtained at $1.6 \mathrm{~K}$. Moments are located on special positions $8 a$ for $\mathrm{Ni} / \mathrm{Cu}$ ions and $16 d$ for $\mathrm{Cr}$ ions of the orthorhombic space group $F d d d$. The positional parameters for oxygen are given in Table II.

\begin{tabular}{|c|c|c|c|c|}
\hline$\overline{\text { Sample }}$ & Type & $\boldsymbol{k}$ & $\mathrm{NiCr}_{2} \mathrm{O}_{4}$ & $\mathrm{Ni}_{0.98} \mathrm{Cu}_{0.02} \mathrm{Cr}_{2} \mathrm{O}_{4}$ \\
\hline $\begin{array}{l}\mu_{x}(\mathrm{Ni} / \mathrm{Cu})\left[\mu_{\mathrm{B}}\right] \\
\mu_{x}(\mathrm{Cr}) \\
{\left[\mu_{\mathrm{B}}\right]}\end{array}$ & FI & $(000)$ & $\begin{array}{l}-2.2(1) \\
1.1(1)\end{array}$ & $\begin{array}{l}-1.9(1) \\
1.0(1)\end{array}$ \\
\hline$\mu_{z}(\mathrm{Cr})\left[\mu_{\mathrm{B}}\right]$ & $\mathrm{AF}, S L-I$ & $(0,0,1)$ & $1.9(1)$ & $1.9(1)$ \\
\hline$\mu_{z}(\mathrm{Cr})\left[\mu_{\mathrm{B}}\right]$ & $\mathrm{AF}, S L-I$ & $\left(0,0, k_{z}\right)^{*}$ & $1.6(1)$ & $1.4(1)$ \\
\hline$\mu_{z}(\mathrm{Cr})\left[\mu_{\mathrm{B}}\right]$ & $\mathrm{AF}, S L-I I$ & $(1 / 2,1 / 2,1 / 2)$ & $0.5(1)$ & $1.8(1)$ \\
\hline$\mu_{\mathrm{tot}}(\mathrm{Cr})\left[\mu_{\mathrm{B}}\right]$ & $S L-I$ & & $2.7(2)$ & $2.6(2)$ \\
\hline$\mu_{\text {tot }}(\mathrm{Cr})\left[\mu_{\mathrm{B}}\right]$ & $S L-I I$ & & $1.2(2)$ & $2.1(1)$ \\
\hline
\end{tabular}




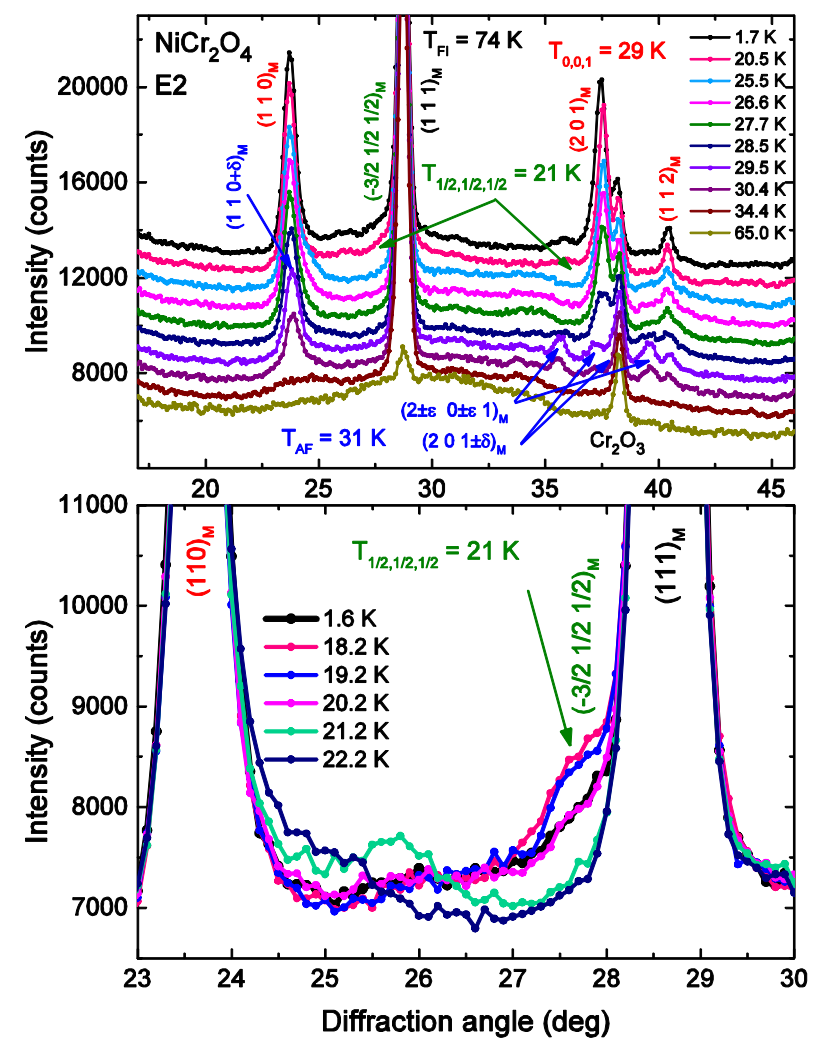

FIG. 1. Neutron powder diffraction patterns of $\mathrm{NiCr}_{2} \mathrm{O}_{4}$ at different temperatures. At $1.7 \mathrm{~K} \mathrm{a}$ ferrimagnetic phase $(\boldsymbol{k}=0)$ coexists with two antiferromagnetic phases with the vectors $\boldsymbol{k}=$ $(0,0,1)$ and $\boldsymbol{k}=(1 / 2,1 / 2,1 / 2)$. At $29 \mathrm{~K}$ a commensurate-incommensurate transition is observed, where one find two coexisting phases with the vectors $\boldsymbol{k}=(0,0,1-\delta)$ and $\boldsymbol{k}=(-\varepsilon, \varepsilon, 1)(\delta=0.05$ and $\varepsilon \cong 1 / 8)$. In the lower part of the diagram the temperature dependence of the reflection $I\left(1 \frac{1 / 2}{2}\right.$ $-1 / 2 \frac{1 / 2}{)_{\mathrm{M}}}$ is presented in more detail. In the range between 15 and $18 \mathrm{~K}$ the intensity of this reflection reaches the maximum intensity before it disappears at $21 \mathrm{~K}$. The magnetic reflection of the impurity $\mathrm{Cr}_{2} \mathrm{O}_{3}$ occurs at $38.3^{\circ}$. At $21.2 \mathrm{~K}$ a single reflection occurs at the angle 25.7 deg, which may be magnetic. However, it was impossible to attribute this reflection to another magnetic phase. 


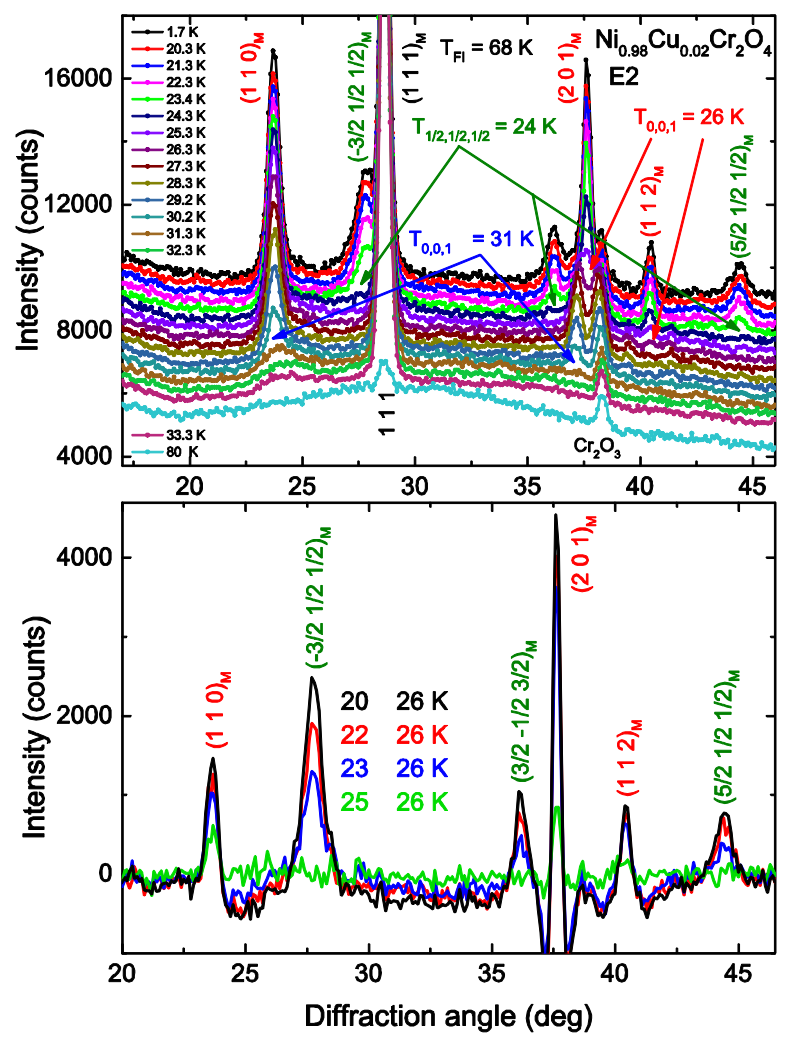

FIG. 2. Neutron powder diffraction patterns of $\mathrm{Ni}_{0.98} \mathrm{Cu}_{0.02} \mathrm{Cr}_{2} \mathrm{O}_{4}$ at different temperatures. At 1.7 a ferrimagnetic phase $(\boldsymbol{k}=0)$ coexists with two antiferromagnetic phases with the vectors $\boldsymbol{k}=(0,0,1)$ and $\boldsymbol{k}=(1 / 2,1 / 2,1 / 2)$ and an incommensurate one $\boldsymbol{k}=(0,0,1-\delta)(\delta=0.09)$. At $24 \mathrm{~K}$ a magnetic transition is observed, where one finds a complete change into the incommensurate phases with the vector $\boldsymbol{k}=(0,0,1-\delta)(\delta=0.09)$. In the lower part of the diagram the difference patterns with respect to a measurement at $26 \mathrm{~K}$ are plotted showing the temperature dependence of the $(1 / 2,1 / 2,1 / 2)$ phase. The two negative reflections result from the subtraction of the $26 \mathrm{~K}$ pattern containing the incommensurate $(0,0,1 \pm \delta)$ phase which vanishes below $25 \mathrm{~K}$. The magnetic reflection of the impurity $\mathrm{Cr}_{2} \mathrm{O}_{3}$ occurs at $38.3^{\circ}$. 


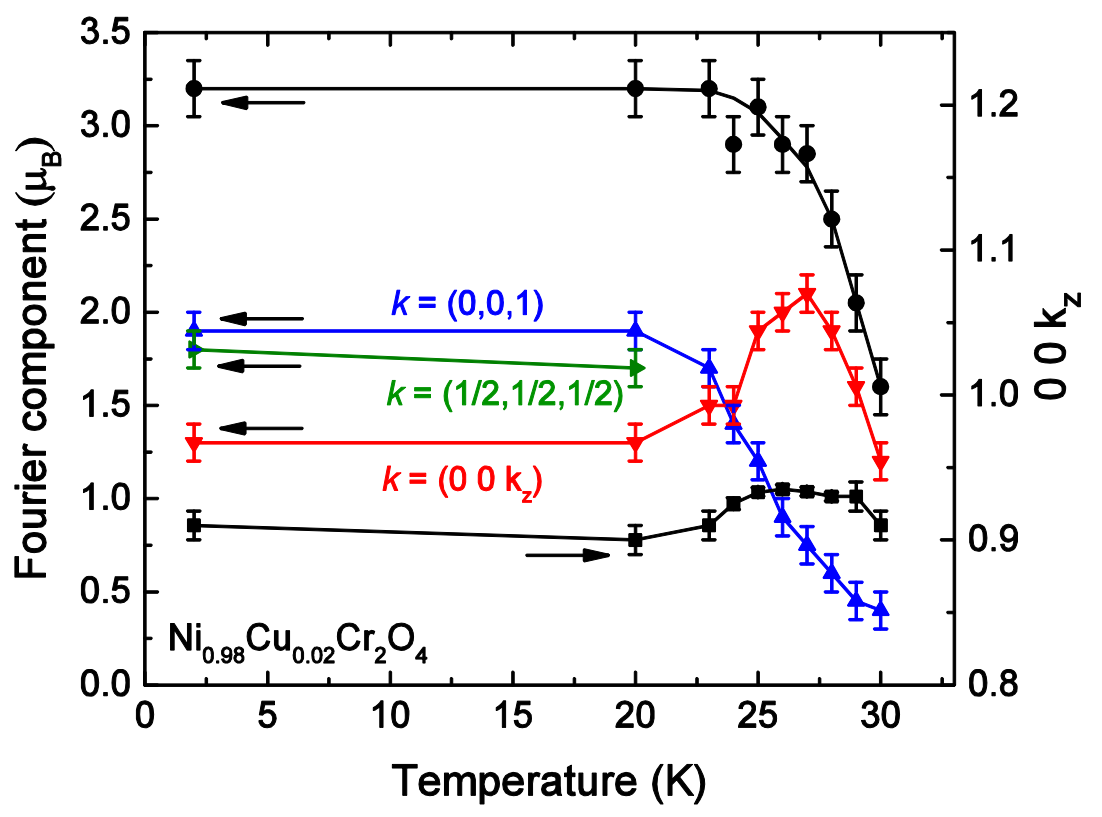

FIG. 3. Temperature dependence of the Fourier components of moments for different magnetic phases (left ordinate). The total moment values are shown in form of black filled circles. The right-hand ordinate represents the variation of the $k_{z}$ component of the $\left(0,0, k_{z}\right)$ phase (black).

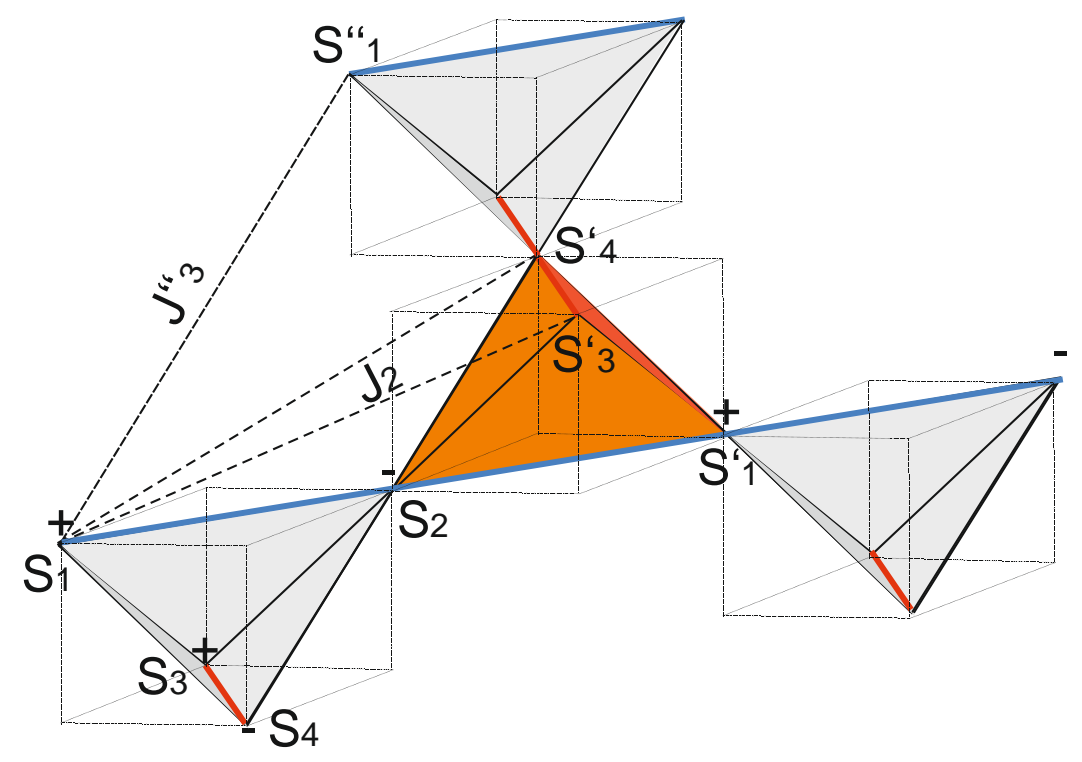

FIG. 4. Four corner-sharing $\mathrm{Cr}_{4}$ tetrahedra of the pyrochlore structure. Blue and red lines are diagonals in the $a_{1} a_{2}$ plane indicating the two $S L-I$ and $S L-I I$, respectively. Magnetic bonds between second and third neighbors are labelled $J_{2}$ and $J_{3}$ ". 


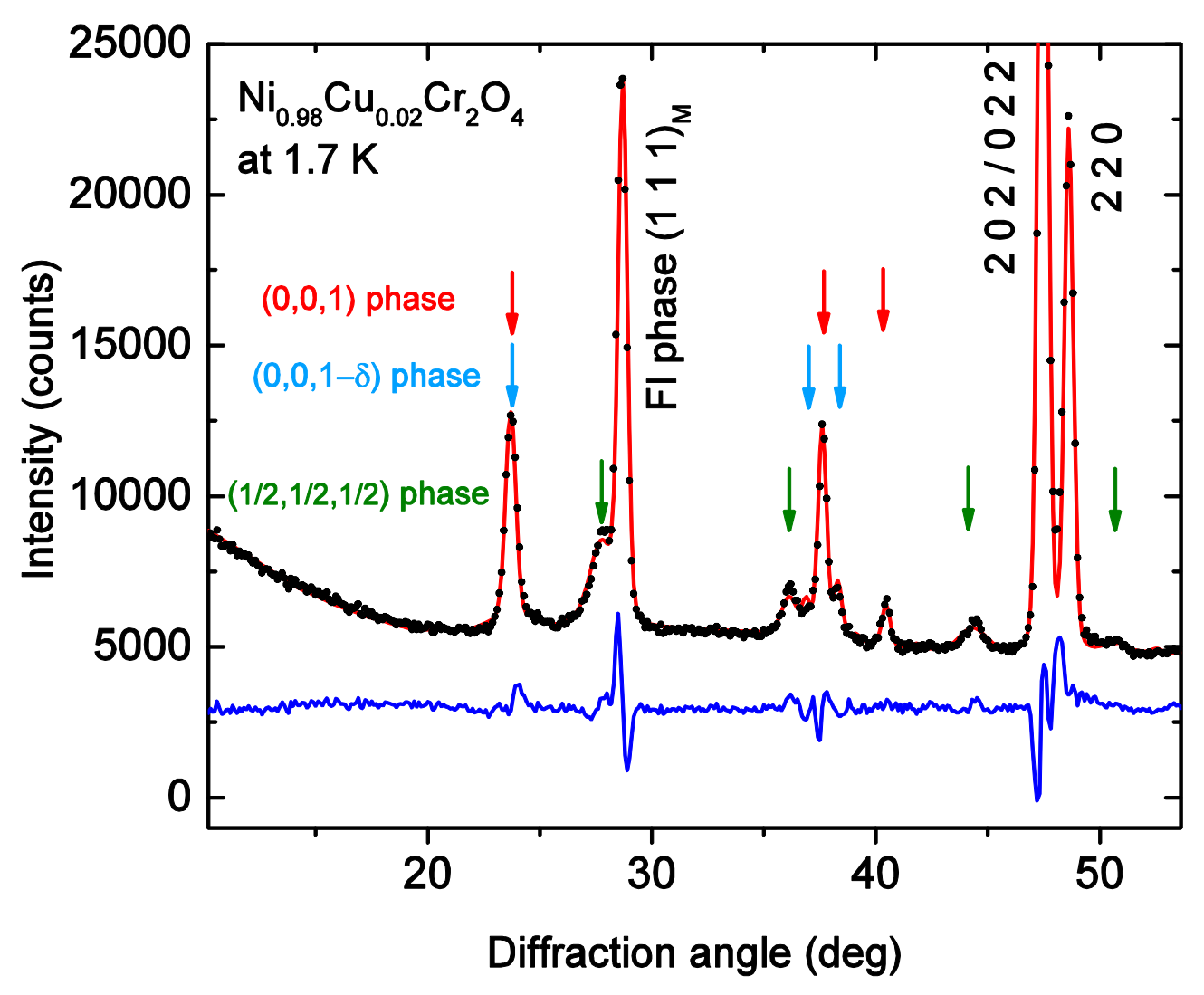

FIG. 5. (Color online) Refinement for $\mathrm{Ni}_{.98} \mathrm{Cu}_{.02} \mathrm{Cr}_{2} \mathrm{O}_{4}$. Observed (black) and calculated pattern (red) are shown together with difference plot. The ferrimagnetic $\boldsymbol{k}=0$ phase (FI) coexists with the two antiferromagnetic $(0,0,1)$ and $(1 / 2,1 / 2,1 / 2)$ and an incommensurate $(0,0,1-\delta)$ phase with $\delta=0.09$. 


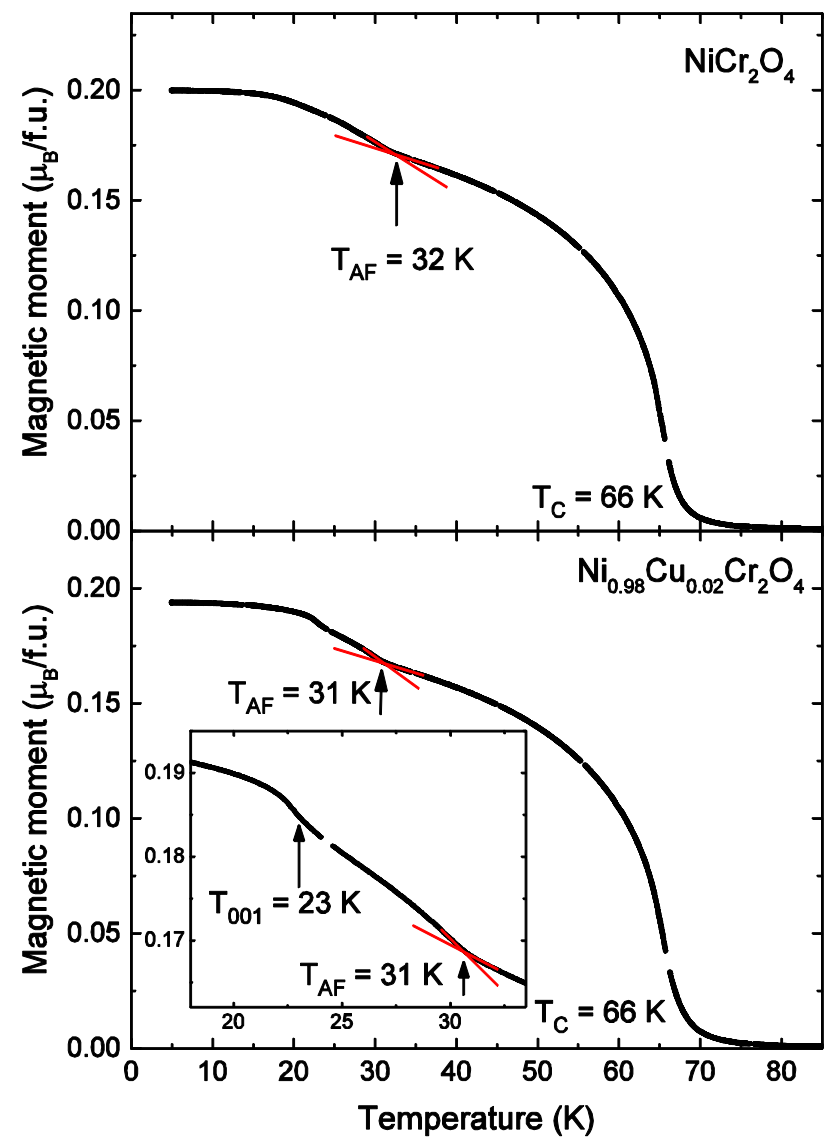

FIG. 6. (Color online) Temperature dependence of the magnetic moment of $\mathrm{NiCr}_{2} \mathrm{O}_{4}$ and $\mathrm{Ni}_{0.98} \mathrm{Cu}_{0.02} \mathrm{Cr}_{2} \mathrm{O}_{4}$ as determined from magnetization measurements with an applied field of $0.05 \mathrm{~T}$. Ferrimagnetic ordering sets in for both compounds below $T_{\mathrm{C}}=66 \mathrm{~K}$. A first anomaly occurs for both compounds at $T_{\mathrm{AF}}=32$ and $31 \mathrm{~K}$, indicating the onset of antiferromagnetic ordering. For $\mathrm{Ni}_{0.98} \mathrm{Cu}_{0.02} \mathrm{Cr}_{2} \mathrm{O}_{4}$ the curve shows a second anomaly at $23 \mathrm{~K}$ with the onset of the $(1 / 2,1 / 2,1 / 2)$ phase. 


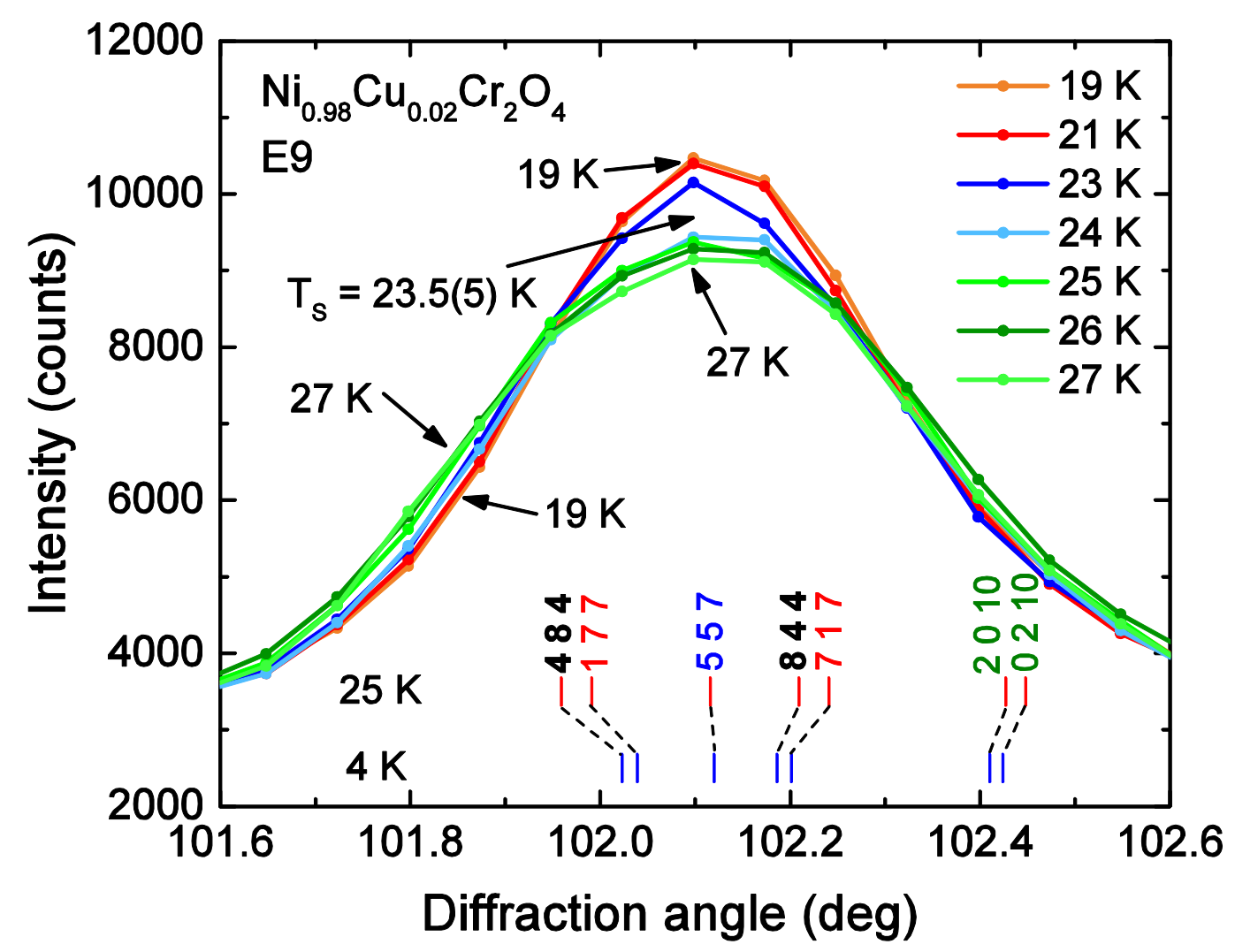

FIG. 7. (Color online) Temperature dependence of the strong peak observed at the diffraction angle $102.2^{\circ}$ measured on E9. A spontaneous peak broadening sets in at $T_{\mathrm{S}}=24 \mathrm{~K}$, which can be ascribed to the shifts of the Bragg reflections 844 and 484 , which dominate the peak intensity: 844 (100), 484 (95), 177 (14), 717 (13), 2010 and 0210 (6), 557 (5) [h kl (Intensity, normalized to 100 for 844 )]. The shifts of the $h k l$ positions are obtained from the Rietveld refinements using data sets collected at 4 and $25 \mathrm{~K}$. 

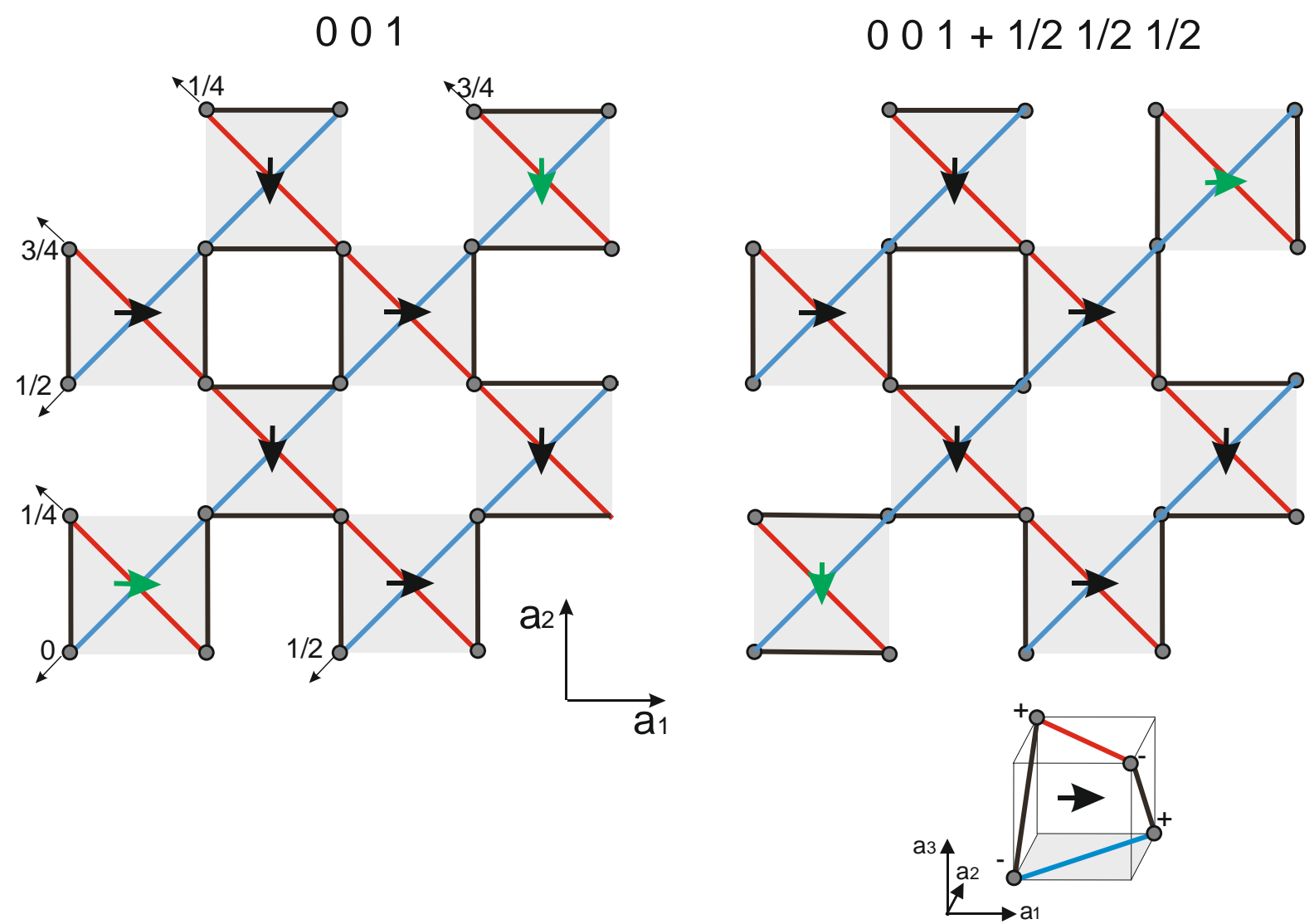

FIG. 8. (Color online) Magnetic bonds for structures with $(0,0,1)$ phase or with $(0,0,1) \&$ $(1 / 2,1 / 2,1 / 2)$ phases projected to the $a_{1} a_{2}$ plane. Diagonal arrows and numbers specify the fractional coordinate along $a_{3}$ for the chains. Solid lines indicate AF bonds. Red (blue) lines connect spins on $S L-I$ (SL-II). Black lines show AF bonds between spins belonging to different sublattices on a $\mathrm{Cr}_{4}$-tetrahedron inscribed in a cuboid. Bold arrows within $\mathrm{Cr}_{4}$ tetrahedra mark elongation in the $a_{1} a_{2}$ plane according to magnetoelastic effect. Green arrows indicate differing local distortions between the two structures. 